\title{
A new fabrication method for hierarchical truss materials with millimeter-scale struts
}

\author{
Qianqian $\mathrm{Wu}^{\mathrm{a}}$, Li Ma ${ }^{\mathrm{a}}$, Ying Gao ${ }^{\mathrm{a}}$, Jian Xiong ${ }^{\mathrm{a}, \mathrm{b} *}$ \\ ${ }^{a}$ Center for Composite Materials and Structures, Harbin Institute of Technology \\ Harbin 150001, PR China \\ ${ }^{\mathrm{b}}$ National Key Laboratory of Science and Technology on Advanced Composites in Special Environments
}

Harbin 150001, PR China

\begin{abstract}
Hierarchical truss materials with millimeter-scale struts have been fabricated using a three-dimensional printing and interlocking method. The limitation designs of hierarchical truss materials have been found in this short letter to show the imperfection of hierarchical materials. The analytical models and experimental work have been conducted to investigate the restriction mechanism of the hierarchical design. Polymer hierarchical truss materials with low relative densities $(0.66 \%$ to $2.67 \%)$ have been fabricated for compressive tests. Euler buckling of hierarchical and first order truss materials has been observed in our experiments. Our conclusions are very useful for accurate comprehension of hierarchical construction and designing ultra-lightweight hierarchical truss materials with high specific strength at low density to avoid the deficiencies of this structural configuration.
\end{abstract}

Keywords: Hierarchical materials; Porous materials; Elastic properties; Three dimensional printing; Interlocking method.

\section{Introduction}

Honeycomb, foam and integrated woven materials are commonly used as core materials and they are one type of bionic design based on natural materials [1-4]. Efforts to develop lightweight and multifunctional structures have attracted extensive interests all over the world, such as man-made foam and lattice materials having been fabricated and their mechanical behaviors investigated [5-8]. Many natural and man-made materials also exhibit structure on more than one scale, in some materials, the structural elements themselves have smaller scale structures. This structural hierarchy can play a major part in determining the bulk material properties. Lattice truss [9-10], honeycomb [11-14] and corrugated [15-16] geometries with centimeter-scale elements were considered, and it was shown that a hierarchical material could be used in the design of their structural elements which, for a given compressive strength, are much lighter than first order (solid) elements.

Almost all of the previous papers have shown the superiority of a hierarchical design for lightweight sandwich structures due to larger size struts (centimeter-scale), while the relevant design limitations of such types of structures remains an open issue because of the fabrication difficulty. Hierarchical truss materials with small (millimeter-scale) struts cannot be fabricated and the hierarchical design cannot be fully implemented to provide it with higher structural efficiency. In our paper, a three-dimensional printing and interlocking method are used to manufacture this type of structures on a small scale. The analytical calculations have been derived to show the new limitation of hierarchical truss materials and experiments have been conducted. No similar

\footnotetext{
${ }^{*}$ Corresponding author, Tel.:+86 451 86402376; fax: +86 45186402376.

E-mail address: jx@hit.edu.cn (Professor Jian Xiong)
} 
research work has been found in the literature.

\section{Theoretical work of hierarchical truss materials}

Two possible failure modes will be considered in our analytical models: Euler buckling and crushing for first order truss materials. Five possible failure modes (core face wrinkling, core face crushing, corrugated wall buckling, corrugated wall crushing and core buckling) have been considered for the hierarchical truss materials. For the buckling modes, there are two possible bending directions due to different bending moments.

2.1 First order truss materials

\subsubsection{Euler buckling}

There are two directions for strut buckling and according the method in our previous paper [17], the analytical equations can be derived as:

$$
\bar{\sigma}_{b}=\frac{8 \xi \pi^{2}(E I)_{e}}{l^{\prime 4} \cos ^{2} \omega^{\prime}}
$$

where $\xi=\frac{12(E I) \cos ^{2}\left(\omega^{\prime}\right)+(E A) l^{\prime 2} \sin ^{2}\left(\omega^{\prime}\right)}{(E A) l^{\prime 2} \sin \left(\omega^{\prime}\right)}, \quad$ and $(E I)_{e}$ is the bending stiffness of the first order pyramidal strut. The struts length, $l^{\prime}$, and the inclination angle between the struts and the base of the unit cell, $\omega^{\prime}$, of first order truss materials, are defined in Fig. 1(a). EI and EA denote the strut's bending stiffness and compressive stiffness of first order pyramidal lattice structure respectively.

\subsubsection{Crushing}

The nominal compressive crushing strength of a truss core strut is calculated by:

$$
\bar{\sigma}_{f}=\frac{2 \xi \sigma_{f} A_{m}}{l^{\prime 2} \cos ^{2} \omega^{\prime}}
$$

where $A_{m}$ is the area of cross section, $\sigma_{f}$ is the failure strength of parent polymer materials.

\subsection{Hierarchical truss materials}

\subsubsection{Core face wrinkling}

This failure occurs between the adhesive points of corrugated cores and the face sheet of the core will wrinkle under the compressive loading:

$$
\bar{\sigma}=\frac{\xi(1+\eta) \pi^{2} E b t_{1}^{3}}{3 l_{1}^{2} l_{2}^{2} \cos ^{2}\left(\omega_{1}\right) \cos ^{2}\left(\omega_{2}\right)}
$$

where $\eta=\frac{t_{2}^{3} \sin ^{2}\left(\omega_{2}\right) \cos \left(\omega_{2}\right)+t_{2} l_{2}^{2} \cos ^{3}\left(\omega_{2}\right)}{2 t_{1} l_{2}^{2}}, \quad$ and $E$ is the stiffness of parent polymer materials. The geometric design variables are shown in Fig. 1(b) and the geometric dimensions have been given in Table 1.

\subsubsection{Core face crushing}

The corresponding theoretical prediction formula of the hierarchical truss core crushing can be expressed as:

$$
\bar{\sigma}=\frac{4 \xi(1+\eta) b t_{1} \sigma_{f}}{l_{1}^{2} \cos ^{2}\left(\omega_{1}\right)}
$$




\subsubsection{Corrugated wall buckling}

The theoretical prediction for corrugated wall buckling is given below as:

$$
\bar{\sigma}=\frac{2 \xi \lambda_{c} \pi^{2} E b t_{2}^{3}}{3 l_{1}^{2} l_{2}^{2} \cos ^{2}\left(\omega_{1}\right)}
$$

where $\lambda_{c}=\cos \left(\omega_{2}\right)+\frac{t_{2}^{2} \sin ^{2}\left(\omega_{2}\right)}{l_{2}^{2} \cos \left(\omega_{2}\right)}+\frac{2 t_{1}}{t_{2} \cos ^{2}\left(\omega_{2}\right)}$.

\subsubsection{Corrugated wall crushing}

The effective compressive strength of the hierarchical pyramidal core associated with the onset of corrugated wall crushing can be calculated from:

$$
\bar{\sigma}=\frac{2 \xi \lambda_{c} b t_{2} \sigma_{f}}{l_{1}^{2} \cos ^{2}\left(\omega_{1}\right)}
$$

\subsubsection{Core buckling}

The nominal compressive strength of a pyramidal core due to the elastic buckling of its struts can be given by:

$$
\bar{\sigma}=\frac{8 \xi \pi^{2}(E I)_{e}}{l_{1}^{4} \cos ^{2}\left(\omega_{1}\right)}
$$

where $(E I)_{e}=(E I)_{c e}^{12}$ for buckling in 1-2 direction, and $(E I)_{e}=(E I)_{c e}^{13}$ for buckling in 1-3 direction, the ends of struts are assumed to be fixed as boundary conditions $(\mu=0.5)$. The expressions of $(E I)_{c e}^{12}$ and $(E I)_{c e}^{13}$ are as follows:

$$
\begin{gathered}
(E I)_{c e}^{12}=2(E I)_{m}^{12}+\left(4 \cos \omega_{2}-3 \cos ^{5} \omega_{2}\right)(E I)_{p}^{12}+\frac{1}{4} l^{2} \sin ^{2} \omega_{2} \cos ^{3} \omega_{2}(E A)_{p} \\
(E I)_{e}^{13}=2(E I)_{m}^{13}+(E I)_{p}^{13}
\end{gathered}
$$

where $(E A)_{p}$ is the compressive stiffness of corrugated truss, $(E A)_{p}=E b t_{2} ;(E A)_{m}$ is the compressive stiffness of the second order face sheet, $(E A)_{m}=E b t_{1} ;(E I)_{p}^{12}$ and $(E I)_{p}^{13}$ denote the bending stiffness of corrugated truss in the 1-2 direction and that in the 1-3 direction respectively, $(E I)_{p}^{12}=E b t_{2}^{3} / 12$ and $(E I)_{p}^{13}=E t_{2} b^{3} / 12 ;(E I)_{m}^{12}$ and $(E I)_{m}^{13}$ are the bending stiffness of the second order face sheet in the 1-2 direction and that in the 1-3 direction, $(E I)_{m}^{12}=E b t_{1}^{3} / 12$ and $(E I)_{m}^{13}=E t_{1} b^{3} / 12$.

\section{Experimental}

\subsection{Materials and fabrication}

The full name of PVC is polyvinyl chloride and polyvinyl chloride is a thermoplastic resin that was used in our printing process. It is widely prepared to be a film, pipe, sheet metal and energy-saving material with low price and mass production. The mechanical properties of parent materials were tested and the value of stiffness and strength are approximately $1.5 \mathrm{GPa}$ and $26.25 \mathrm{MPa}$, respectively, according to the tension experiments. Our polymer hierarchical truss materials were made by combining three-dimensional printing and interlocking methods. The dimensions of the hierarchical trusses with millimeter-scale struts and face sheets with slots were 
firstly designed using CAD software, and then the geometrical models were inserted into the computer for three dimensional printing. PVC films with the adhesive material can be used to form the truss struts and face sheets layer by layer, finally, the rest of the materials, besides the struts, will be removed easily due to the lack of adhesive outside the designed dimensions. The polyvinyl chloride material is suitable for printing process without obvious defects and then the millimeter-scale struts can be fabricated with high quality. Fig. 1(c-g) illustrates the fabrication process of polymer hierarchical truss materials, which consists of four steps. Firstly, two types of interlocking pieces are fabricated using three dimensional printing (SD300Pro 3D printer). The laminated 3D printing utilizes a plastic sheet which shall be cut in accordance with the cross-sectional data and the plastic sheets are stacked layer by layer to form a three-dimensional solid structure subsequently. Besides that, the operating temperature of the SD300Pro 3D printer is controlled at between $18^{\circ}$ and $30^{\circ}$. However, in this more usual fusion deposition modelling process, the thermoplastics are heated past their glass transition temperature and then deposited by an extrusion head. The Solido SD300Pro printer in which the modeling material used is PVC and the thickness of each layer is $0.168 \mathrm{~mm}$. The machine precision is controlled within $0.1 \mathrm{~mm}$ and the sound level of the running machine is up to $65 \mathrm{db}$. The dimensions of these complex pieces (shown in Fig. 1(c)) are approximately $1 \mathrm{~mm}$ and they have not previously been fabricated because of their small scale. Secondly, Piece I and Piece II were mechanically interlocked with each other to construct the hierarchical truss core in the form shown in Fig. 1(d). Thirdly, upper and bottom face sheets with cross-shaped slots were made using three-dimensional printing (see Fig. 1(e)). These slots play a role in orienting the hierarchical truss struts. Finally, the hierarchical truss core was assembled with the face sheets in the slots using the normal temperature curing liquid epoxy adhesive, as shown in Fig. 1(f). Fig. 1(g) shows the pictures of specimens with and without the face sheets.

\subsection{Experimental setup}

The applied load was measured by the load cell in the INSTRON 5569, while a laser extensometer (Epsilon) was used to measure the nominal compressive strain. The compression tests were carried out in the quasi-static regime with a nominal displacement rate of $0.5 \mathrm{~mm} / \mathrm{min}$ at room temperature.

\section{Results and discussion}

Fig. 1 displays the hierarchical truss materials with multiple cells and the manufacturing progress of the hierarchical truss materials has been addressed in this paper. In order to capture the failure modes and failure positions of the hierarchical truss materials accurately, the single cells of structural hierarchy (Fig. 2) are manufactured firstly and then carried out under compressive loading.

\subsection{Mechanical responses}

The compressive stress-strain response of specimens with three different relative densities is plotted in Fig. 2(a). The compressive stress is defined as the external force $(F)$ divided by the bottom surface area of a unit cell in specimens and the compressive strain is defined as the vertical downward displacement during the process of the applied compressive force divided by the initial height of the overall structure $\left(H+2 t_{f}\right)$. Both structures have similar compressive behaviors. The initial linear response of the structures is followed by a nonlinear regime due to the progressive failure of struts. As the stress reached the maximum value, Euler buckling occurred as shown in Fig. 2(b). Subsequently, with increasing strain, a series of local buckling events occurred between the points of attachment to the truss cores. As the test continues, the stress decreases with the 
increasing strain until the final destruction of the structure.

\subsection{Failure mechanism}

Both Euler buckling and crushing have been found in our experiments. For the specimens with relative densities of $0.66 \%$ to $2.67 \%$, all of the hierarchical trusses failed in the 1-3 direction and showed weaker strength than a first order truss. Previous studies have demonstrated that the strength of the hierarchical design is higher because all of their specimens failed in the 1-2 direction which means that buckling occurred in the thickness direction. Fig. 2(b) shows the relevant failure mode observed in the out-of-plane compression tests. Euler buckling has a significant effect on the overall behavior of hierarchical and first order structures. The failure mode of the truss core changed from Euler buckling to crushing with increasing compression loads. The theoretical values of strength under compression loads are based on the analytical calculations provided in Section 2. The measured results for the compressive strength of hierarchical and first order structures at three different relative densities are summarized in Table 2 and compared with the analytical calculations. The compressive strength value of the hierarchical truss core bending in the 1-3 direction is lower than that bending in the 1-2 direction. According to the equations (7), (8) and (9), the hierarchical truss buckles in the 1-2 direction under the condition when the width of first order truss is greater than $8.7 \mathrm{~mm}$. In this case, the anti-buckling property of hierarchical truss materials can be significantly improved by comparison with that of first order truss materials. The analytically predicted values are slightly higher than the experimental results. This is because defects existing in the three-dimensional printing and even in the interlocking process are not considered in the theoretical calculations. Good agreement is observed between the measurements and predictions for Euler buckling.

\section{Conclusions}

Polymer hierarchical truss materials with millimeter-scale struts were manufactured using a three-dimensional printing and interlocking method. The compressive performance of hierarchical and first order trusses were further investigated by theoretical and experimental work. When the hierarchical truss buckles in the 1-3 direction, the strength of hierarchical truss is much weaker than the first order truss. The design of the hierarchical trusses must ensure that the entire specimen fails in the thick dimension so that their improved performance can be realized. In general, good agreement between theoretical predictions and measurements has been observed. Our conclusions are helpful for improving hierarchical materials and designs with higher strength at low density.

\section{Acknowledgements}

The present work was supported by the National Science Foundation of China under grant Nos. 11302060. JX also gratefully acknowledges supported by Program for Outstanding Young Scholars in Harbin Institute of Technology.

\section{References}

[1] S.D. Pan, L.Z. Wu, Y.G. Sun, Z.G. Zhou. Mat. Lett. 62 (3) (2008) 523-526.

[2] O. Huber, H. Klaus. Mat. Lett. 63 (13-14) (2009) 1117-1120.

[3] D.S. Li, C.Q. Zhao, N. Jiang, L. Jiang. Mat. Lett. 148 (2015) 103-105.

[4] E.W. Andrews, T. Tashi, S. Jun. Mat. Lett. 62 (28) (2008) 4319-4321.

[5] C.S. Zhu, C. Guo, Z.D. Dai. Mat. Lett. 94 (2013) 189-192.

[6] M.P. Staiger, I. Kolbeinsson, N.T. Kirkland, T. Nguyen, G. Dias, T.B.F. Woodfield. Mat. Lett. 64 (23) (2010) 2572-2574. 
[7] Y.S. Lee, B.H. Yeon, S.K. Hyun, K.J. Kang. Mater. Lett. 89 (2012) 279-282.

[8] B.K. Lee, K.J. Kang. Scripta. Mater. 60 (6) (2009) 391-394.

[9] S. Yin, L.Z. Wu, S.R. Nutt. Mater. Design. 56 (2014) 731-739.

[10] F.F. Sun, C.L. Lai, H.L. Fan, D.N. Fang. Mech. Mater. 97 (2016) 164-183.

[11] D. Mousanezhad, B. Haghpanah, R. Ghosh, A.M. Hamouda, H. Nayeb-Hashemi, A. Vaziri. Theor. Appl. Mech. Lett. 6 (2) (2016) 81-96.

[12] A. Ajdari, B.H. Jahromi, J. Papadopoulos, H. Nayeb-Hashemi, A. Vaziri. Int. J. Solids. Struct. 49 (11-12) (2012) 1413-1419.

[13] D. Mousanezhad, S. Babaee, H. Ebrahimi, R. Ghosh, A.S. Hamouda, K. Bertoldi, A. Vaziri. Sci. Rep. 5 (2015) 18306.

[14] J.X. Qiao, C.Q. Chen. Int. J. Solids. Struct. 85-86 (2016) 57-66.

[15] S. Kazemahvazi, D. Zenkert. Compos. Sci. Technol. 69 (7-8) (2009) 913-919.

[16] M.N. Velea, C. Schneider, S. Lache. Mater. Design. 102 (2016) 313-320.

[17] J. Xiong, L. Ma, L.Z. Wu, B. Wang, A. Vaziri. Compos. Struct. 92 (11) (2010) 2695-2702.

\section{Figures}

Fig. 1. Sketch of unit cell and fabrication processes of polymer hierarchical truss materials: (a) unit cell of first order, (b) unit cell of hierarchical truss materials. (c)two types of pieces made by three dimensional printing for interlocking, (d) interlocking topologies, (e)upper and bottom face sheets made by three dimensional printing, (f) assembling with face sheets and core parts, (g) pictures of specimens without and with face sheets.

Fig.2. (a) Stress-strain curves, (b) Euler buckling failure modes for specimen with three different relative densities: $0.66 \%, 1.32 \%$ and $2.67 \%$.

Table 1 The geometric dimensions for the hierarchical truss materials and the first order counterparts with three different densities.

Table 2 Calculated and measured results for the compressive strength of hierarchical and first order truss materials at three different relative densities. The failure modes in this table are abbreviated: $\mathrm{EB}=$ Euler buckling; $\mathrm{FC}=$ face sheet crushing; $\mathrm{CFW}=$ core face wrinkling; $\mathrm{CFC}=$ core face crushing; $\mathrm{CWB}=$ corrugated wall buckling; $\mathrm{CWC}=$ corrugated wall crushing; Analyt. $=$ analytical; Exp. $=$ experiment. 

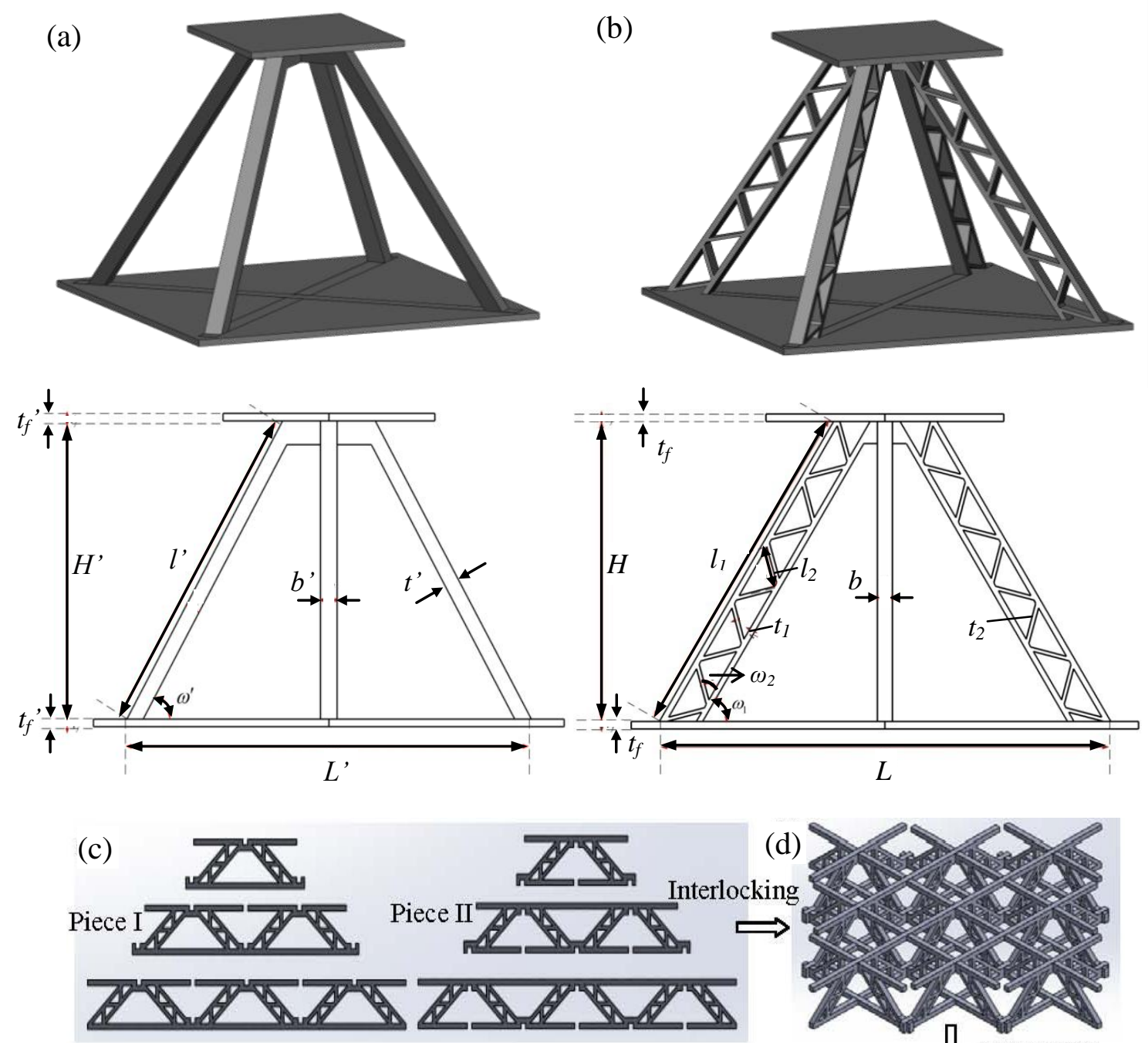

(e)

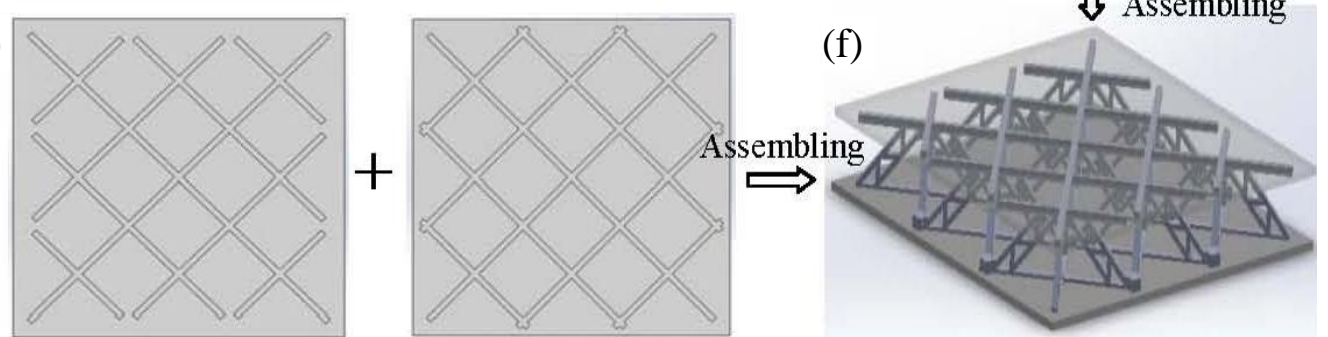

(g)

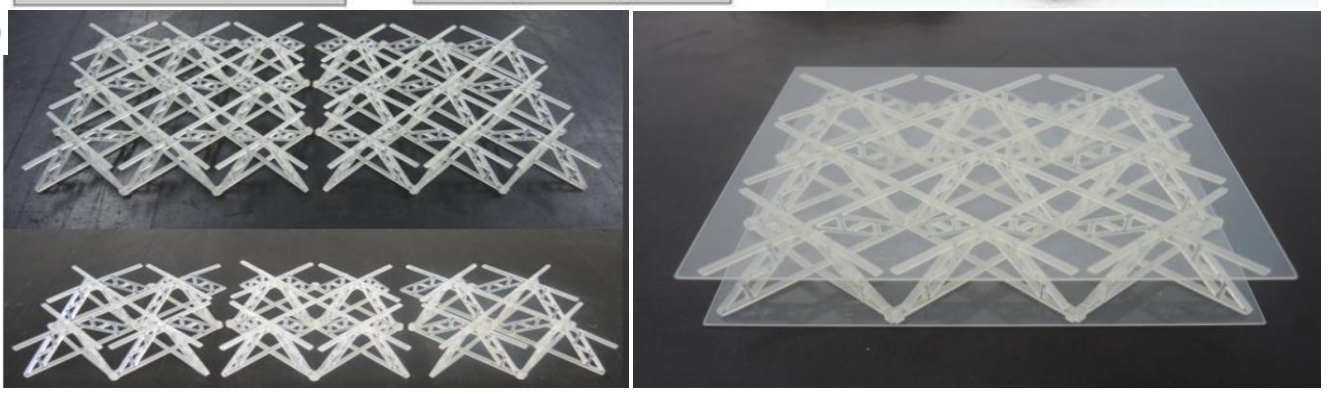

Figure 1 


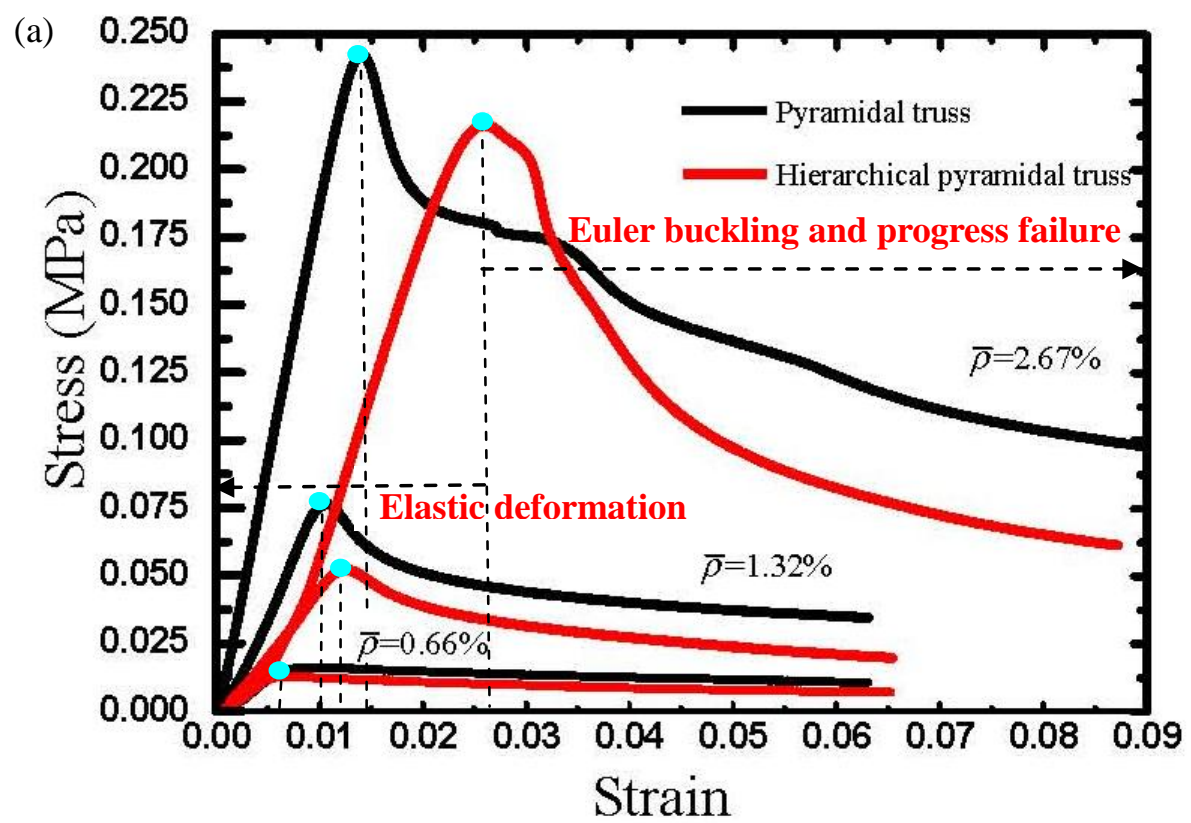

(b)
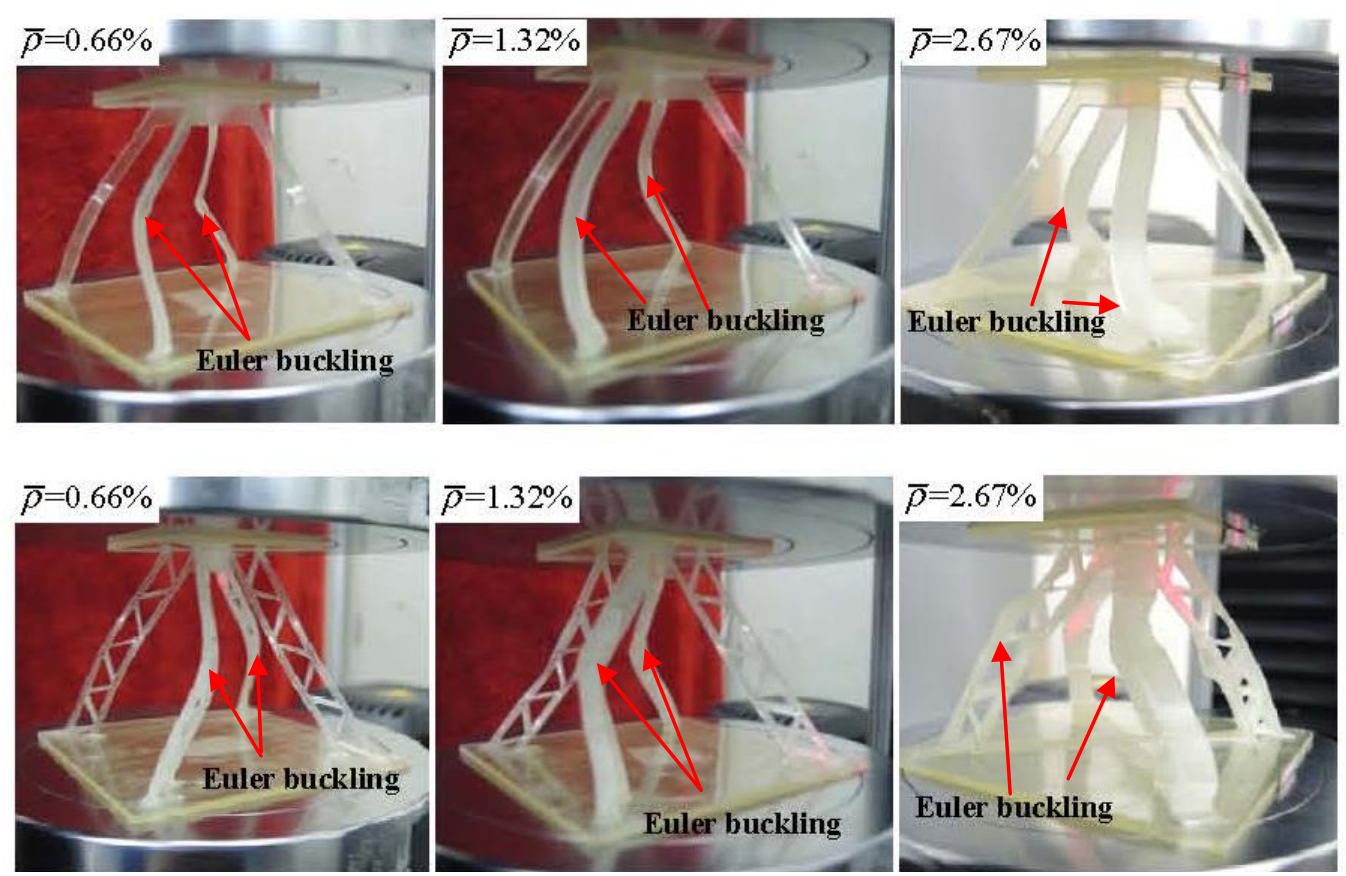

Figure 2 
Table 1 The geometric dimensions for the hierarchical truss materials and the first order counterparts at three different densities.

\begin{tabular}{|c|c|c|c|c|c|c|c|c|c|c|c|}
\hline \multirow[t]{2}{*}{$1^{\text {st }}$ order } & \multicolumn{2}{|c|}{$\bar{\rho}^{\prime}(\%)$} & $\omega^{\prime}\left(^{\circ}\right)$ & \multicolumn{2}{|c|}{$t^{\prime}(m m)$} & $l^{\prime}(m m)$ & $b^{\prime}(m m)$ & \multicolumn{3}{|c|}{$L^{\prime}(m m)$} & $H^{\prime}(\mathrm{mm})$ \\
\hline & \multicolumn{2}{|c|}{0.66} & 60 & \multicolumn{2}{|c|}{4} & 74.81 & 2 & \multicolumn{3}{|c|}{105.91} & 64.79 \\
\hline \multirow{2}{*}{$\begin{array}{c}2^{\text {st }} \\
\text { order }\end{array}$} & $\bar{\rho}(\%)$ & $\omega_{1}\left({ }^{\circ}\right)$ & $\omega_{2}\left({ }^{\circ}\right)$ & $t_{1}(\mathrm{~mm})$ & $t_{2}(\mathrm{~mm})$ & $l_{1}(\mathrm{~mm})$ & $l_{2}(\mathrm{~mm})$ & $b(\mathrm{~mm})$ & $n$ & $L(\mathrm{~mm})$ & $H(\mathrm{~mm})$ \\
\hline & 0.66 & 60 & 45 & 1.5 & 1 & 74.81 & 9.9 & 2 & 4 & 105.91 & 64.79 \\
\hline \multirow[t]{2}{*}{$1^{\text {st }}$ order } & \multicolumn{2}{|c|}{$\bar{\rho}^{\prime}(\%)$} & $\omega^{\prime}\left({ }^{\circ}\right)$ & \multicolumn{2}{|c|}{$t^{\prime}(\mathrm{mm})$} & $l^{\prime}(\mathrm{mm})$ & $b^{\prime}(m m)$ & \multicolumn{3}{|c|}{$L^{\prime}(m m)$} & $H^{\prime}(m m)$ \\
\hline & \multicolumn{2}{|c|}{1.32} & 60 & \multicolumn{2}{|c|}{4} & 74.81 & 4 & \multicolumn{3}{|c|}{105.91} & 64.79 \\
\hline \multirow{2}{*}{$\begin{array}{c}2^{\text {st }} \\
\text { order }\end{array}$} & $\bar{\rho}(\%)$ & $\omega_{1}\left({ }^{\circ}\right)$ & $\omega_{2}\left(^{\circ}\right)$ & $t_{1}(\mathrm{~mm})$ & $t_{2}(\mathrm{~mm})$ & $l_{1}(\mathrm{~mm})$ & $l_{2}(\mathrm{~mm})$ & $b(\mathrm{~mm})$ & $n$ & $L(\mathrm{~mm})$ & $H(\mathrm{~mm})$ \\
\hline & 1.32 & 60 & 45 & 1.5 & 1 & 74.81 & 9.9 & 4 & 4 & 105.91 & 64.79 \\
\hline \multirow[t]{2}{*}{$1^{\text {st }}$ order } & \multicolumn{2}{|c|}{$\bar{\rho}^{\prime}(\%)$} & $\omega^{\prime}\left({ }^{\circ}\right)$ & \multicolumn{2}{|c|}{$t^{\prime}(\mathrm{mm})$} & $l^{\prime}(m m)$ & $b^{\prime}(m m)$ & \multicolumn{3}{|c|}{$L^{\prime}(m m)$} & $H^{\prime}(m m)$ \\
\hline & \multicolumn{2}{|c|}{2.67} & 60 & \multicolumn{2}{|c|}{4} & 74.81 & 8 & \multicolumn{3}{|c|}{105.91} & 64.79 \\
\hline \multirow{2}{*}{$\begin{array}{c}2^{\text {st }} \\
\text { order }\end{array}$} & $\bar{\rho}(\%)$ & $\omega_{1}\left({ }^{\circ}\right)$ & $\omega_{2}\left(^{\circ}\right)$ & $t_{1}(\mathrm{~mm})$ & $t_{2}(\mathrm{~mm})$ & $l_{1}(\mathrm{~mm})$ & $l_{2}(\mathrm{~mm})$ & $b(\mathrm{~mm})$ & $n$ & $L(\mathrm{~mm})$ & $H(\mathrm{~mm})$ \\
\hline & 2.67 & 60 & 45 & 1.5 & 1 & 74.81 & 9.9 & 8 & 4 & 105.91 & 64.79 \\
\hline
\end{tabular}


Table 2 Calculated and measured results for the compressive strength of hierarchical and first order truss materials at three different relative densities. The failure modes in this table are abbreviated: $\mathrm{EB}=$ Euler buckling; $\mathrm{FC}=$ face sheet crushing; $\mathrm{CFW}=$ core face wrinkling; $\mathrm{CFC}=$ core face crushing; $\mathrm{CWB}=$ corrugated wall buckling; $\mathrm{CWC}=$ corrugated wall crushing; Analyt. $=$ analytical; Exp. $=$ experiment.

\begin{tabular}{|c|c|c|c|c|}
\hline Structures & Relative & Analyt.(MPa) & Exp.(MPa) & Failure modes \\
\hline \multirow{3}{*}{ First order } & \multirow{11}{*}{$0.66 \%$} & $\begin{array}{ll}\mathrm{EB}(12) & 0.070\end{array}$ & \multirow{3}{*}{0.016} & \multirow{3}{*}{$\mathrm{EB}(13)$} \\
\hline & & EB(13) & & \\
\hline & & $\begin{array}{ll}\mathrm{FC} & 0.130\end{array}$ & & \\
\hline \multirow{8}{*}{ Hierarchical } & & CFW(12) 5.166 & \multirow{8}{*}{0.013} & \multirow{8}{*}{$\mathrm{EB}(13)$} \\
\hline & & CFW(13) 9.183 & & \\
\hline & & CFC $\quad 0.110$ & & \\
\hline & & $\mathrm{CWB}(12)$ & & \\
\hline & & $\begin{array}{ll}\text { CWB(13) } & 1.690\end{array}$ & & \\
\hline & & CWC & & \\
\hline & & $\mathrm{EB}(12)$ & & \\
\hline & & EB(13) & & \\
\hline \multirow{3}{*}{ First order } & \multirow{11}{*}{$1.32 \%$} & $\mathrm{~EB}(12) \quad 0.14$ & \multirow{3}{*}{0.075} & \multirow{3}{*}{$\mathrm{EB}(13)$} \\
\hline & & $\begin{array}{ll}\text { EB(13) } & 0.14\end{array}$ & & \\
\hline & & FC $\quad 0.26$ & & \\
\hline \multirow{8}{*}{ Hierarchical } & & CFW(12) 10.331 & \multirow{8}{*}{0.050} & \multirow{8}{*}{$\mathrm{EB}(13)$} \\
\hline & & CFW(13) 73.465 & & \\
\hline & & $\begin{array}{ll}\text { CFC } & 0.220\end{array}$ & & \\
\hline & & \multirow{2}{*}{$\begin{array}{lr}\text { CWB(12) } & 0.845 \\
\text { CWB(13) } & 13.520\end{array}$} & & \\
\hline & & & & \\
\hline & & CWC $\quad 0.441$ & & \\
\hline & & $\mathrm{EB}(12)$ & & \\
\hline & & $\mathrm{EB}(13)$ & & \\
\hline \multirow{3}{*}{ First order } & \multirow{11}{*}{$2.67 \%$} & $\begin{array}{ll}\mathrm{EB}(12) & 0.279 \\
\end{array}$ & \multirow{3}{*}{0.250} & \multirow{3}{*}{$\mathrm{EB}(12)$} \\
\hline & & $\begin{array}{ll}\mathrm{EB}(13) & 1.117\end{array}$ & & \\
\hline & & $\begin{array}{ll}\text { FC } & 0.519\end{array}$ & & \\
\hline \multirow{8}{*}{ Hierarchical } & & CFW(12) 20.662 & \multirow{8}{*}{0.215} & \multirow{8}{*}{$\begin{array}{c}\mathrm{EB}(13) \\
\mathrm{CFC}\end{array}$} \\
\hline & & CFW(13) 587.720 & & \\
\hline & & $\begin{array}{ll}\text { CFC } & 0.440\end{array}$ & & \\
\hline & & \multirow{2}{*}{$\begin{array}{lc}\text { CWB(12) } & 1.690 \\
\text { CWB(13) } & 108.16\end{array}$} & & \\
\hline & & & & \\
\hline & & $\begin{array}{ll}\text { CWC } & 0.881 \\
\end{array}$ & & \\
\hline & & $\mathrm{EB}(12)$ & & \\
\hline & & $\mathrm{EB}(13)$ & & \\
\hline
\end{tabular}

